\title{
Friedrich Nietzsche und der Midi
}

„Viele verborgene Flecke und Höhen aus der Landschaft Nizza’s sind mir durch unvergessliche Augenblicke geweiht“ (EH, KSA 6, 341) hält Friedrich Nietzsche in seinem entstandenen 1888 autobiographischen Text Ecce Homo fest. „[j]ene entscheidende Partie, welche den Titel ,von alten und neuen Tafeln` trägt, wurde im beschwerlichsten Aufsteigen von der Station zu dem wunderbaren maurischen Felsenneste Eza gedichtet, die Muskel-Behendheit war bei mir immer am grössten, wenn die schöpferische Kraft am reichsten floss“ (ebd.). Zu diesem zentralen Kapitel seines Werkes Also sprach Zarathustra, dessen dritter und vierter Teil während der Winteraufenthalte 1883/1884 und 1884/1885 entstanden ist, hat Nizza und seine Umgebung den deutschen Philosophen inspiriert. Von 1883 bis 1888 verbringt Nietzsche jeden Winter in Nizza, das er mal zur „Cosmopolis“ (Nr. 650, KGB III/3, 120) stilisiert, mal als „unausstehlich! - unwürdig! - großstädtisch-lärmend - stupid!“ (Nr. 569, KGB III/3, 5) brandmarkt. Sils Maria im Engadin im Sommer und Nizza im Winter, nach langem Suchen hat der stets gesundheitlich stark angeschlagene Philosoph - entweder plagt ihn der Kopf, der Magen oder der Rücken, fast immer schmerzen den Halbblinden die Augen - 1883 seine „Heimaten“ gefunden. „Im Jahr 220 vollkommen reine Tage, das entschied zuletzt: die Wirkung dieser herrlichen Lichtfülle auf mich sehr gequälten Sterblichen (und so oft Sterbenslustigen) grenzt an's Wunderbare“ (Nr. 474, KGB III/1, 457) schreibt er im Dezember 1883 an seinen treuen Weggefährten, den Komponisten Heinrich Köselitz alias Peter Gast. Der Midi öffnet bei dem Wanderer, der beim Morgenspaziergang Aphorismen notiert, ${ }^{1}$ Schleusen der Kreativität.

Der Raum, die Riviera/Côte d'Azur, wird Schauplatz einer Serie von rauschhaften Schreibwellen, die in höheren Lagen, in Rapallo und Sils Maria, begonnen haben, sich aber dann in den ,Sanctus Januarius'-Ereignissen, weniger in Genua als in Nizza, fortsetzen. In einer seltsamen Mischung aus Askese und Ekstase im Schreibakt entstehen so zentrale Werke wie Zarathustra, Jenseits von Gut und Böse und Die fröhliche Wissenschaft. Es sind Wintererlebnisse in trockener sonniger Kälte. Dabei stehen das Pensionselend und der Schaffensrausch in einem eigentümlichen Gegensatz. Die Hochstimmung findet ihre Entsprechung in hochgestimmten Texten.

Bekanntlich flieht Nietzsche das deutsche Flachland und das entsprechend flachgeistige neugegründete Deutsche Kaiserreich zugunsten von Gebirge und Meer. So mag sein Aufenthalt an der Côte d'Azur als Bewegung eines Konvertiten erscheinen, der mit fliegenden Fahnen zur französischen Kultur überläuft. Indes hat Nietzsche eine höchst selektive Wahrnehmung von Frankreich. Er bewundert das Frankreich

1 Vgl. Paul Lanzky, Erinnerungen an Friedrich Nietzsche, in: Allgemeine Zeitung vom 16. 10. 1893. 
der Renaissance, insbesondere die Selbstbeobachtung von Michel de Montaigne, die klassische Epoche, hier die psychologische Analyse der Moralisten, den stolzen Einzelgänger Stendhal und die l'art pour l'art-Position von Charles Baudelaire, schließlich in zeitgenössischem Kontext Paul Bourget. Durchgängig verteidigt er das aristokratische Vornehmheitsideal und die psychologischen Einsichten, die ohne die bierselige deutsche Innerlichkeit möglich werden. Das fromme Frankreich - vor allem das egalitäre Frankreich der Dritten Republik - blendet er hingegen aus, es ist mit seinem Denken völlig unvereinbar.

Der genius loci, den Nietzsche sich im Midi erwählt, entspricht einer gemischten Randlage. Das gerade französisch gewordene Nizza, mit einer zum Teil italienisch sprechenden, zum Teil französisch sprechenden Bevölkerung ist durch Mehrfachzugehörigkeiten gekennzeichnet. Es stellt nicht nur eine Mischzone zwischen Italien und Frankreich dar. Mit seinem Meeressaum, dem Midi, dem Mittag, öffnet sich das vornehme psychologische Frankreich zu einem entgrenzenden Mittelmeer, auf dessen anderen Seite Afrika liegt.

Nietzsche schneidet sich nun eine sehr schmale klimatische Zone aus diesen Raumvektoren heraus. Bereits etwas weiter östlich, in Genua, bekommt ihm das Klima weniger gut. Er will die Küste von Nizza bis Saint-Raphaël zu Fuß erkunden. Es ist eine Zone, die die Steigerung der Lebenskräfte verspricht. Die Grenzen rahmen einen Ort ein, an dem er gesünder wird. Sehr konkret heißt dies, dass Nizza der Ort ist, an dem Nietzsche weniger Kopfschmerzen hat, sich weniger übergibt, weniger Ekel empfindet, weniger an Widerwärtigem würgt. Wie kaum ein anderer Denker behauptet Nietzsche, dass sich die Ortserfahrung selbst in der Lebensführung und im Werk niederschlägt. Die Lebensphilosophie gewinnt hier eine räumliche Dimension. Nietzsche spricht dem Raum die Macht zu, das Geheimnis der ewigen Wiederkehr zum Ausdruck zu bringen.

Die Frage ist, ob Nietzsches Schreibformen des ,Mittags', die ihm im Midi gelingen, die Aneignung seines Denkens durch die französische Philosophie und Kunst erleichtern. Mittler ist Nietzsche weniger durch seine Aufnahme französischer Denkfiguren als durch eine lebensweltliche Vermittlung seiner lebensphilosophischen Philosophie, aber auch durch sein Fortwirken nach seinem Tode in Frankreich.

\section{Der französische Nietzsche}

Seit den neunziger Jahren des 19. Jahrhunderts bis heute dauert in Frankreich eine selten unterbrochene intensive Beschäftigung mit Nietzsche an. ${ }^{2}$ Jüngste Beispiele sind die Februar-Ausgabe 2009 der französischen Literaturzeitschrift Lire, ${ }^{3}$ die den

2 Vgl. Werner Hamacher, Echolos, in: Ders. (Hg.), Nietzsche aus Frankreich, Berlin, Wien 2003, 11.

3 Vgl. Nietzsches Philosophe de la Vie, in: Lire, 2 (2009). 
deutschen Philosophen zum Titelthema gemacht hat, ohne dass ein bestimmtes Jubiläum dies bedingt hätte, und die Erzählung Nietzsche à Nice von Patrick Mauriès, ${ }^{4}$ die ihm den 1888 im Alter von nur 34 Jahren im Midi verstorbenen Philosophen JeanMarie Guyau zur Seite stellt. Das ungebrochene Interesse an dem 1900 in geistiger Umnachtung Gestorbenen versucht Alfons Reckermann damit zu erklären, dass Nietzsche in Frankreich keineswegs ausschließlich der Universitätsphilosophie gehöre: „Der französische Nietzsche verweigert sich der Rolle eines philosophischakademischen Problemlösers. Sein Denken eröffnet vielmehr einen konfliktgeladenen Bedeutungsraum, den die Philosophie betreten muss, wenn sie in der Gegenwart mehr als musealen Rang besitzen oder mehr als die registrierende Rolle eines Beobachters oder Interpreten der Debatten anderer Wissenschaften übernehmen will. “5

Die Rezeption des Werkes des deutschen Philosophen in Frankreich reißt nicht ab. ${ }^{6}$ Schon zu Lebzeiten Nietzsches, allerdings nach dem Zusammenbruch in Turin im Jahre 1889, machen sich der Begründer der modernen französischen Germanistik, Henri Lichtenberger, und Henri Albert, Journalist beim Mercure de France, für die Verbreitung seines Werkes stark. Letzterer übersetzt zunächst Also sprach Zarathustra, später die meisten Bücher Nietzsches ins Französische. Zu der ungewöhnlichen Wirkung des Zarathustra-Autors in Frankreich äußert sich bereits 1899 André Gide. $^{7}$ Während für Gide der ,Immoralismus‘ im Vordergrund steht, der die Intensität des Lebens steigert, hebt nach dem Ersten Weltkrieg der französische Germanist Charles Andler den Abstand Nietzsches vom Kaiserreich hervor. Geneviève Bianquis überträgt seine noch nicht übersetzten Bücher ins Französische, allerdings auch das von Elisabeth Förster-Nietzsche fabrizierte Machwerk Der Wille zur Macht.

In den dreißiger Jahren findet Nietzsches Denken eine widersprüchliche Aufnahme. Nietzsches zweiter französischer Augenblick ${ }^{8}$ und damit der entscheidende Schritt in Richtung eines französischen Nietzscheanismus liegt zweifelsohne in der Arbeit des Collège de Sociologie im Jahre 1937. Die Soziologen Georges Bataille und Roger Caillois, der Ethnologe Michel Leiris, der Schriftsteller Pierre Klossowski und der Philosophieprofessor Jean Wahl, der als erster Nietzsches Philosophie an der Sorbonne lehrt, entwickeln seine Theorie der Entfremdung des Individuums weiter, um ökonomische, soziale und politische Fragen zu diskutieren. Es geht in diesen

4 Patrick Mauriès, Nietzsche à Nice, Paris 2009.

5 Alfons Reckermann, Lesarten der Philosophie Nietzsches, Berlin, New York 2003, 90.

6 Vgl. zum Thema Nietzsche und Nizza: Yves Séméria, Les saisons niçoises de Frédéric Nietzsche. Les paysages de l'esprit 1883-1888, Nizza 2001; Christian Schärf, Palmen, Mittag, Ewigkeit, in: Frankfurter Allgemeine Zeitung vom 2. 5. 2009; Ders., Friedrich Nietzsche in Nizza, in: Magali Laure Nieradka, Denise Specht (Hg.), Fremdkörper? Aspekte der Geisteswissenschaften in der Auslandsgermanistik und im DaF-Unterricht, Münster 2009.

7 Vgl. Werner Hamacher, Echolos, 7.

8 Vgl. Vincent Descombes, Le moment français de Nietzsche, in: Alain Boyer, André Comte-Sponville, Vincent Descombes (Hg.), Pourquoi nous ne sommes pas nietzschéens, Paris 1991, 101. 
Texten der Franzosen um die Entfesselung von Lebenskräften, die erst mit der Entthronung des sich autonom glaubenden Subjekts möglich werden. Das Zerbrechen von Identität findet Entsprechungen in der Form der Nietzsche-Texte, wie etwa der Literaturtheoretiker Maurice Blanchot in seiner Studie über das Fragment bei Nietzsche demonstriert.

Freilich gibt es in Frankreich wie in Deutschland eine andere Linie der NietzscheRezeption, die in trübes Gewässer führt. Nietzsches Aufklärungs-, Demokratie- und Sozialismuskritik wird vor allem von extremistischen Intellektuellen, beispielsweise Thierry Maulnier, Journalist der Zeitschrift Action Française, oder bei dem Kollaborateur Pierre Drieu la Rochelle, von 1940 bis 1943 Leiter der Nouvelle Revue Française, aufgegriffen. ${ }^{9}$

Mit den Philosophen Gilles Deleuze und Michel Foucault, welche die Tradition eines französischen Nietzscheanismus weiterführen, kommt es, so Vincent Descombes, in den sechziger und siebziger Jahren zu Nietzsches drittem französischen Augenblick. ${ }^{10}$ Auch Jacques Derrida, Philippe Lacoue-Labarthe und Jean-Luc Nancy formulieren ihre Kritik des Logozentrismus anhand von Nietzsche-Studien. Es gibt jetzt, wie Werner Hamacher es nennt, einen Nietzsche aus Frankreich ${ }^{11}$ und einen, wie Jacques Le Rider sagt, Nietzsche in Frankreich. ${ }^{12}$ Dies wiederum bewirkt eine Auseinandersetzung der deutschen Wissenschaft mit dem deutschen Philosophen, dessen Euvre seit der Pervertierung durch die Nationalsozialisten in Deutschland verpönt gewesen ist. Der seit den achtziger Jahren einsetzende Rücktransfer des (französisierten) Nietzsche nach Deutschland löst widersprüchliche Reaktionen aus: heftigen Widerstand vor allem bei Jürgen Habermas und Manfred Frank; zustimmende Aneignung bei Gert Mattenklott, Gerd Bergfleth und Peter Sloterdijk.

Lange Zeit habe man seinen gewaltigen Einfluss auf die französische Kultur durch ein präexistenten Nietzscheanismus zu erklären versucht, so Mazzino Montinari. Dieser Ansatz müsse heutzutage revidiert werden. Nicht die französische Literatur und Philosophie sei auf Nietzsche vorbereitet gewesen, sondern Nietzsche selber habe sich durch seine umfassende Kenntnis und Assimilation der französischen Kultur des fin de siècle für seine Wirkung in Frankreich präpariert. ${ }^{13}$ So heißt es in dem Kapitel Warum ich so gute Bücher schreibe im Ecce Homo: „In Wien, in St. Peterburg, in Stockholm, in Kopenhagen, in Paris und New-York. Überall bin ich entdeckt: ich bin es nicht in Europa's Flachland Deutschland [...] Deutsch denken, deutsch fühlen - ich kann Alles, aber das geht über meine Kräfte ... Mein alter Lehrer Ritschl behauptete

9 Vgl. Jacques Le Rider, Nietzsche in Frankreich, München 1997; Ders., Nietzsche en France, Paris 1999. 10 Vgl. Vincent Descombes, Le moment français de Nietzsche, 123.

11 Vgl. Fußnote 2.

12 Vgl. Fußnote 9.

13 Vgl. Mazzino Montinari, Nietzsche heute, zit. nach: Jacques Le Rider, Nietzsche in Frankreich, München 1997, 7. 
sogar, ich concipirte selbst noch meine philologischen Abhandlungen wie ein Pariser romancier absurd spannend“ (EH, KSA 6, 301).

Nietzsche aus Frankreich gäbe es nicht ohne Nietzsche in Frankreich, deshalb kehren wir nun nach Frankreich und nach Nizza zurück. Die Frage ist, in welcher Weise der französische Kontext das Werk Nietzsches selbst bestimmt.

\section{Nietzsche in Frankreich und Nizza}

Zwar fällt sein erster Frankreichaufenthalt bereits auf das Jahr 1870, doch wird dieser für sein Leben und Werk keine große Rolle spielen. Im Deutsch-Französischen Krieg von 1870/1871 meldet sich der fünfundzwanzigjährige Basler Professor für Altphilologie als Freiwilliger. Der deutsche Patriot, der zu diesem Zeitpunkt voller Misstrauen gegen das moderne Frankreich und ein erklärter Feind der französischen Sozialisten und Republikaner ist, ${ }^{14}$ arbeitet vom 25. August bis zum 3. September 1870 als Pfleger im Lazarett im Nordelsass und in Lothringen. Während eines Verwundetentransports zurück nach Deutschland erkrankt er nach nur zehn Tagen Dienst an Rachendiphtherie und Ruhr und wird von der weiteren Kriegsteilnahme entbunden. ${ }^{15}$ Auch während seiner Schul- und Studienzeit ist er mit Frankreich und der französischen Kultur nur wenig in Kontakt gekommen. Zwischen 1850 und 1858 hat er in der Bürgerschule und in der Domschule zu Naumburg eine rein altphilologische Erziehung erfahren. Erst in der Landesschule in Pforta, wo er 1864 das Abitur macht, hat er Französischunterricht. ${ }^{16}$ In seinem ersten Bonner Studienjahr plant er eine Parisreise, die sich allerdings zerschlägt. ${ }^{17}$ Als Professor in Basel kommt der gebürtige Sachse allmählich mit der Romania in Verbindung. Der erste wirkliche Mittler in punkto Frankreich ist für ihn der Schweizer Kulturhistoriker Jacob Burckhardt. Seine universale, die Antike wie die Romania umfassende Bildung ist für den Altphilologen ein Ansporn, sich intensiver mit Frankreich auseinander zu setzen. ${ }^{18}$ Burckhardt wirkt auf Nietzsche romanisch und Nietzsche wiederum auf Burckhardt teutonisch. ${ }^{19}$ Doch es wird noch über ein Jahrzehnt dauern, bis Nietzsche zum ersten Mal wirklich französischen Boden betreten wird.

14 Vgl. ders., ebd., 7.

15 Vgl. Raymond J. Benders, Stephan Oettermann, Hauke Reich, Sibylle Spiegel (Hg.), Friedrich Nietzsche. Chronik in Bildern und Texten, München 2000, $227 \mathrm{f}$.

16 Vgl. Beatrix Bludau, Frankreich im Werk Nietzsches. Geschichte und Kritik einer Einflussthese, Bonn 1979, 113.

17 Vgl. dies. ebd., 114.

18 Vgl. dies., ebd., 116.

19 Vgl. Edgar Salin, Vom deutschen Verhängnis. Gespräch an der Zeitenwende: Burckhardt - Nietzsche, Hamburg 1959, 46. 
Im Jahre 1879 gibt Nietzsche seinen Lehrstuhl für Altphilologie in Basel auf, weil seine körperlichen Leiden eine Fortführung seiner Tätigkeit nicht erlauben. Fortan führt der heimatlose Philosoph eine Reiseexistenz. Dem eifrigen Briefeschreiber verdanken wir durch seine Korrespondenz eine genaue Beschreibung seiner Stationen und Lebensumstände. Venedig, Genua, Rapallo und Sizilien sind nur einige seiner Ziele. Immer wieder treibt es ihn weiter, weil ihm das Klima nicht bekommt, bis er die französische Riviera für sich entdeckt. An Nizza, das erst seit $1870 \mathrm{zu}$ Frankreich gehört, schätzt er allerdings nicht das französische, sondern das italienische Flair. Am 4. Dezember 1883 schreibt er an Köselitz: „Nizza, als französische Stadt, ist mir unleidlich und fast ein Flecken in dieser südländischen Herrlichkeit; aber es ist auch noch eine italiänische Stadt da, im älteren Theil, habe ich mich eingemiehtet, und wenn geredet werden muß, wird italiänisch geredet“ (Nr. 474, KGB III/1, 457). Und am selben Tag an seine Schwester Elisabeth und an seine Mutter: „Die lärmende elegante Stadt mißfiel mir Anfangs; zuletzt habe ich aber Manches herausgefunden, was für mich übrig stille Wege und italiänische Stadt-Theile, bessere Kost als in Genua und für einen bescheidenen Prinzen, wie ich bin, im Ganzen auch alte Genueser Preise. Es ist eine große Stadt, man kann's haben, wie man will. Das Wichtigste aber ist, daß es keine Kranken-Stadt ist viel zu frisch und windig: während es dieselbe Lichtfülle und Zahl der reinen Tage hat, wie jene Krankenorte, an denen ich nicht gehängt sein möchte“ (Nr. 475, KGB III/1, 458).

Während seines ersten Aufenthalts vom 2. Dezember 1883 bis zum 20. April 1884 wohnt er zuerst im benachbarten Villefranche, bevor er ein Eckzimmer in der rue Ségurane Nummer 38 in der Nähe des Hafens bezieht. Doch Nietzsche hat kein glückliches Händchen bei der Wahl seiner Unterkünfte. Da seine kleine Basler Pension ihm keine großen Sprünge erlaubt, wohnt er meist in billigen kalten Hotels, Pensionszimmern oder Mietwohnungen, in denen er sich blaue Finger holt (Nr. 785, KGB III/5, 3). Der Wiener Physiologe Joseph Paneth, der im Winter 1883 Nietzsches Gegenwart sucht, zeigt sich schockiert über die Behausung des Philosophen: „Sein Zimmerchen ist kahl und unfreundlich und gewiss nicht mit Rücksicht auf Bequemlichkeit sondern auf Billigkeit gewählt worden; es hat nicht einmal einen Ofen (!), keinen Teppich und sieht gar nicht hübsch aus und es war eine eisige Kälte dort, während ich darin war. [...] Es wäre mir grässlich, in einem solchen Loch stecken zu müssen, wie Nietzsche, in einem solchen freddo cane.“20

Wenig später zieht Nietzsche in die von einer gewissen Frau Mendschel betriebene Villa Mazzoleni, rue du Châteauneuf 39 im Vallon St. Philippe am anderen Ende von Nizza. Darauf wechselt er in die Pension de Genève in der petite rue St. Etienne (heute rue Rossini), wo er bis zum Ende seines ersten Nizza-Winters wohnt. Die Pension de Genève wird künftig Nietzsches Anlaufstelle und Stammquartier in Nizza.

20 Richard Frank Krummel (Hg.), Joseph Paneth über seine Begegnung mit Nietzsche in der Zarathustra-Zeit, in: Nietzsche Studien, 17 (1988), 479 f. 
Auch wenn er dort nicht wohnt, isst er hier häufig zu Abend. In den vier folgenden Wintern kommt er außer in der Pension de Genève in der rue St. François de Paule Nummer 26 und in der Villa Speranza in der rue des Ponchettes Nummer 29 (heute 17) unter. Zwar überzeugt ihn Menton während seines zweiten Aufenthalts an der Riviera (vom 9. November 1884 bis zum 8. April 1885), denn es sei „stiller, großartiger, alles Gebirge und Grün mehr zur Hand“ (Nr. 555, KGB III/1, 556), solle „eine achtungswürdigere Menschheit beherbergen“ (Nr. 556, KGB III/1, 558) und er komme gut mit dem vierten Teil des Zarathustra voran, doch die dortige Einsamkeit und das feuchtere Klima lassen Nizza erneut zu seinem Winterdomizil werden. Immer wieder ist ihm Nizza als „Abklatsch von Paris und großthuerische Halb-Großstadt zuwider; es fehlen Wald, Schatten, Stille in einem kaum glaublichen Maße. Wäre ich vermögend, so würde ich schon anderswo an der riviera leben; aber verhältnismäßig lebe ich hier natürlich am billigsten, weil es die größte Stadt ist“ (Nr. 486, KGB III/1, 475).

Das Mediterrane, das Nietzsche oft „das Afrikanische“ (Nr. 770, KGB III/3, 274) nennt, schätzt er an der blauen Küste. Immer wieder wird der treu ergebene Freund Paul Lanzky, der Nietzsche mit „verehrtester Meister“ (Nr. 364, KGB III/4, 155) anredet, vorgeschickt, um eine neue Bleibe zu erkunden, aber alle Ziele werden von Nietzsche wieder verworfen. Korsika wäre als Zeugungs- und Geburtsstätte Napoleons würdig, allerdings sei die Reise zu beschwerlich. Cannes komme als kleines Nizza nicht in Frage, St. Raphael nicht wegen seiner scharfen Mistralwinde, Monaco nicht, weil es die Mutter der Spielhölle von Monte Carlo sei. ${ }^{21}$ Immer wieder siegt Nizza, Nikaia, die Siegreiche. An Köselitz bringt er am 24. November 1885 zu Papier: „Dieser Tage erquickte es mich zu erfahren, daß diese Stadt, welche ich nicht mehr wechseln und eintauschen darf, in ihrem Namen etwas vom Siege hat. Und wenn Sie hören, wie der Platz heißt, wohinaus mein Fenster schaut (herrliche Bäume, in der Ferne röthliche große Gebäude, das Meer und die schön gewundene baie des anges), nämlich ,Square des Phocéens', so werden Sie vielleicht gleich mir über den ungeheuren Cosmopolitismus dieser Wort-Verbindung lachen - wirklich haben Phoceer einstmals hier sich angesiedelt - aber etwas Siegreiches und Über-Europäisches klingt heraus, etwas sehr Tröstliches, das mir sagt ,hier bist du an deinem Platze ‘...] Ich erwähne endlich, daß man hier billig, sehr billig leben kann, und daß der Ort umfänglich genug ist, um jeden Grad einsiedlerischer Verborgenheit zu gestatten. Die ganz ausgesuchten Dinge der Natur, wie die Waldwege am nächsten Berge, wie die Halbinsel Saint-Jean, hat unsereins für sich; ebenso ist die ganze herrlich-freie Promenade am stark brandenden Meere (ca. dreiviertel Stunde lang -) nur für ein paar Stunden des Tages besucht“ (Nr. 648, KGB III/3, 114 f.).

Während der fünf Winter in Nizza inszeniert Nietzsche für ein deutsches Publikum eine fortschreitende Konversion zur französischen Literatur und zur Romania.

21 Vgl. Paul Lanzky, Friedrich Nietzsche nach persönlichem Umgang, in: Sphinx 18 (1894), 336. 
Charles Andler nennt die achtziger Jahre Nietzsches „französische Periode“.22 Er liest als einzige Zeitung das französische Journal des Débats, dessen philosophischer Mitarbeiter Hippolyte Taine ist. Das Kompliment Taines, Nietzsches Schriften seien „infiniment suggestif“, berührt den deutschen Philosophen zutiefst (Nr. 769, KGB III/3, 272). Er entdeckt die französische Literatur durch Paul Bourgets Essais de la psychologie contemporaine. Er behauptet, die scharfsinnigen Porträts des Schriftstellers würden ihm einen Zugang zur modernen französischen Gegenwartsliteratur vermitteln. Er kenne sich bald in der französischen Literatur besser als in der deutschen aus. Tatsächlich liest er Gustave Flaubert, Théophile Gautier, die Brüder Goncourt, Victor Hugo, Guy de Maupassant, Ernest Renan und allen voran Baudelaire im Original. In der Biographie Förster-Nietzsches kann man lesen, dass sich ihr Bruder schon immer für Frankreich interessiert, dass sich aber die wahre Frankreich-Begeisterung erst in Nizza gezeigt habe: „Vom Winter 1883 an, den er in Nizza verlebte, liebte er Übersetzungen nicht mehr; er hatte sich dort sehr an die französische Sprache gewöhnt, und da sich auch der Zustand seiner Augen bedeutend gebessert hatte, so fing er an, selbst sehr viel französisch zu lesen.“23 Ist auch die Arbeit seiner Schwester als Nachlassverwalterin, Interpretin und Biographin in vielen Punkten fragwürdig, so kann man ihr an dieser Stelle wohl etwas mehr Glauben schenken. Beatrix Bludau zweifelt allerdings an, ob Nietzsche, nach den wenigen Jahren Schulfranzösisch in der Lage gewesen ist, die literarischen Texte ohne Übersetzung zu verstehen. ${ }^{24}$

Der frisch in Zürich promovierten Resa von Schirnhofer gibt Nietzsche jedenfalls die Aufgabe, diese Autoren zu lesen, als sie ihn vom 3. bis 12. April 1884 in Nizza besucht. ${ }^{25}$ Geschickt worden ist ihm die junge Österreicherin von seiner mütterlichen Freundin und Mäzenin Malwida von Meysenbug, die er bei der Grundsteinlegung des Festspielhauses in Bayreuth 1872 kennen gelernt hat. Nietzsche absolviert mit von Schirnhofer ein abwechslungsreiches Tourismusprogramm: Es wird durch die Altstadt Nizzas spaziert, auf der Promenade des Anglais flaniert, auf den Schlossberg gestiegen und auf Nizzas Hausberg, den Mont Boron, gewandert. Dort zaubert Nietzsche einen Vermouth di Torino zum Anstoßen auf ,sein` Nizza, ,seine‘ Entdeckung, mit ,seinem‘ Mistralwind und den Blick auf ,seine‘ Insel Korsika, die natürlich nicht zu sehen ist, hervor. Einen Besuch im Spielcasino in Monte Carlo lehnt die junge Dame ab, dafür lässt sie sich zu einem Stierkampf überreden. Gemäß behördlicher Verordnung dürfen in Nizza allerdings weder Pferde auftreten noch Stiere getötet werden. Das zahme Geplänkel erscheint den beiden germanischen Zuschauern als Karikatur eines Stiergefechts. In der Pause werden Auszüge aus Georges Bizets Oper

22 Jacques Le Rider, Nietzsche in Frankreich, 34.

23 Elisabeth Förster-Nietzsche, Nietzsche und die Franzosen, in: Die Zukunft 26 (1899), 463.

24 Vgl. Beatrix Bludau, Frankreich im Werk Nietzsches, 117.

25 Vgl. Raymond J. Benders, Stephan Oettermann, Hauke Reich, Sibylle Spiegel (Hg.), Friedrich Nietzsche. Chronik in Bildern und Texten, 580. 
Carmen gespielt. Während sie die Musik fehl am Platze findet, wirkt sie auf ihn elektrisierend ${ }^{26}$ ein Phänomen, auf das wir später noch einmal zu sprechen kommen werden. Nach diesen heiteren zehn Tagen überreicht Nietzsche von Schirnhofer seine drei Zarathustra-Bände mit Widmung. Der Abschluss des Manuskripts des dritten Zarathustra wenige Monate zuvor am 18. Januar 1884 hat ihm einen regelrechten Euphorie-Schub gegeben (Nr. 480, KGB III/1, 466 f.), in einem Brief an seinen Verleger steht äußerst von sich selbst überzeugt: „Sie haben jetzt das zukunftreichste Buch in Ihrem Verlag, das es giebt“ (Nr. 485, KGB III/1, 473). Ähnlich überzeugt überreicht er von Schirnhofer sein Werk: „Dann erhob er sich, um sich zu verabschieden, und als wir bei der Türe standen, veränderten sich plötzlich seine Züge. Mit einem starren Ausdruck im Gesicht, scheue Blicke um sich werfend, als würde eine entsetzliche Gefahr drohen, wenn ein Horcher seine Worte hörte, die Hand am Munde den Laut dämpfend, verkündete er mir flüsternd das ,Geheimnis‘, das Zarathustra dem Leben ins Ohr gesagt, worauf im dieses geantwortet hatte: ,Du weißt das, oh Zarathustra?` das weiß niemand. Es lag etwas Bizarres, ja, Unheimliches in der Art, wie Nietzsche die ,ewige Wiederkehr des Gleichen', die ungeheure Tragweite dieser Idee mitteilte.“27

Danach kehrt Nietzsche zu seiner natürlichen Sprechweise zurück. Von Schirnhofer habe das Gefühl gehabt, er habe absichtlich auf dem Instrument ihrer Sensibilität fortissimo gespielt, um ihr das Ungeheure seiner Entdeckung, die ewige Wiederkehr des Gleichen, unvergesslich zu machen. Tatsächlich sei ihr Aufenthalt aber, so Werner Ross, eine Probe für das Empfangen künftiger Jünger gewesen: „Ein scheinbar touristisches, literarisch-gebildetes, in Wahrheit ein „einweihendes“ Programm [...] Der Ovidvers, den er in das Zarathustra-Exemplar Resas eintrug, bedeutete in Nietzsches Sinngebung die Verwandlung des Menschen in ein neues Wesen, und erst recht war die Zarathustra-Lesung als vorbereitende Weihe für den Empfang der Mysterienbotschaft gedacht. “28

Auf der einen Seite sehnt sich Friedrich Nietzsche nach Abgeschiedenheit, nach reiner Natur und verachtet die Gegenwart seiner Mitmenschen wie ein richtiger professorenhafter deutscher Snob. ${ }^{29}$ In der Halbinsel St. Jean Cap Ferrat zwischen Nizza und Monaco meint Lanzky endlich die wahre Wirkungsstätte für den Philosophen gefunden zu haben. Nietzsche selbst schwärmt in einem Brief an seine Schwester und seinen Schwager Bernhard Förster von der Schönheit dieses Fleckchens Erde, das er am Weihnachtstag 1885 erkundet (Nr. 654, KGB III/3, 128 f.). Doch wieder siegt der unruhige Geist der philosophischen Mission: „So blieben wir lange mit der Ausforschung der Halbinsel St. Jean, zwischen Beaulieu und Villefranche, behufs einer

26 Vgl. Hans Lohgerber, Friedrich Nietzsche und Resa von Schirnhofer, in: Zeitschrift für philosophische Forschung 22 (1969), 255.

27 Ebd., ders., 256.

28 Werner Ross, Der ängstliche Adler, München 1984, 698.

29 Vgl. ders., ebd., 711. 
ständigen Ansiedlung beschäftigt. Auf der einen Seite lag Nizza mit den notwendigsten Hülfsmitteln, [...] auf der anderen Seite konnte man ab und zu die klassischen Konzerte von Pasdeloup in Monte Carlo besuchen. Und in der Mitte, gleichsam zu unserer Wohnung gehörend, die ganze Halbinsel St. Jean, vom südlichen Meere umspült, mit Oliven und Reben bewachsen und von vielen bequemen und lauschigen Fußpfaden durchkreuzt. Es war idyllisch schön, aber die ländliche Idylle paßte für Zarathustra nicht! Einige Tage der Ermüdung, eine Sehnsucht nach Ruhe und Stille ließen sie göttlich finden; dann trat wieder der Dämon der übermenschlichen Aufgabe hervor und fand sich wie in gräßlichem Kontrast zu diesem ländlichen Frieden. Der Grund war der: Zarathustra brauchte Schüler, Anhänger, Handlanger, Arbeitsmaterial - das alles bot ihm die Einsamkeit nicht, die er an und für sich nicht ertragen konnte.“30

Zarathustra, das ist Nietzsche. Und Nietzsche braucht Schüler, Anhänger, Handlanger. „Vielleicht gelingt es mir, hier [in Nizza] eine Gesellschaft mir zu begründen, unter der ich nicht ganz der ,Verborgene“ bin“, schreibt er im April 1884 an Franz Overbeck: „Das Clima des littoral provençal gehört auf das Wunderbarste zu meiner Natur; ich hätte den Schlußreim zu meinem Zarathustra nur an dieser Küste dichten können, in der Heimat der ,gaya scienza““ (Nr. 504. KGB III/1, 494 f.). Nietzsche wünscht sich Lanzky, Köselitz und vor allem die beiden Freunde seiner misslungenen ménage à trois, Paul Rée und Lou Andreas-Salomé, denn er leidet unter der Einsamkeit: „Was mir in Nizza fehlt, sind Menschen, die ich liebe und denen man erst nicht alles ,sagen muß‘. Ich bin drei Viertel des Tages ziemlich düster und arbeitsam den Rest lustig oder ,profondément triste', wie es einem einsamen Bär und Philosophen zukommt“ (Nr. 671, KGB III/3, 151). Er will Mittler sein und dafür bedarf es nicht nur der „halkyonischen Meeresstille“ (N. 650, KGB III/3, 119) des Midi, sondern auch des hektischen Nizza, das seine halbblinden Augen bei seinem vierten Aufenthalt (20.10.1886 bis 2. April 1887) bereits auswendig gelernt haben (Nr. 768, KGB III/3, 271). An seine Schwester und seine Mutter schreibt er am 28. November 1884: „Ich will und muß an Nizza festhalten, zum Zweck meiner zukünftigen Colonie, welche mir jetzt möglicher erscheint (ich meine: sympathische Menschen, vor denen ich meine Philosophie doziren kann). So allein, wie hier [in Menton], oder im Engadin, bin ich beständig krank“ (Nr. 560, KGB III/1, 563).

Die geborenen Mittelländer, wie er die Menschen des Midi in seinem Buch Jenseits von Gut und Böse nennt, sind für ihn die ,guten Europäer'. Die Überlegenheit der Franzosen liege darin, dass ,ihr dem Süden periodisch zugewandtes und abgewandtes Temperament, in dem von Zeit zu Zeit das provençalische und ligurische Blut überschäume, sie vor dem schauerlichen nordischen Grau in Grau und der sonnenlosen Begriffs-Gespensterei und Blutarmuth“ bewahre. Genau für diese Menschen habe Bizet Musik gemacht (JGB, KSA 5, 199 f.). Bizet, das ist Nietzsches ,Entdeckung‘. Am 27. 11. 1881 hat Nietzsche in Genua zum ersten Mal Carmen gehört. Während seines

30 Paul Lanzky, Friedrich Nietzsche nach persönlichem Umgang, 336 f. 
fünften Nizza-Winters (23. 10. 1887 bis 2. 4. 1888) erklärt er Köselitz: „Die erste Aufführung von Carmen im großen italiänischen Theater - ein wahres Ereignis für mich: ich habe in diesen 4 Stunden mehr erlebt und begriffen als sonst in 4 Wochen“ (Nr. 964, KGB III/5, 212). Wenige Tage darauf schwärmt Nietzsche gegenüber derselben Person, dass nach einem Abend Musik, er habe Carmen nun viermal hintereinander gehört, ein Morgen voll resoluter Einsichten und Einfälle folge, als ob man in einem natürlicheren Element gebadet hätte (Nr. 976, KGB III/5, 232): „So wie er dem nordischen Pessimisten Schopenhauer als dem Lehrmeister, von dem er sich abstieß, die südliche Philosophie des Lachens und des Tanzes, des Ja zum Leben entgegensetzte, als Triumph und Übertrumpfung, so musste Wagner durch eine südliche, heitere, das Leben feiernde Musik ausgestochen werden. ,Il faut méditerraniser la musique‘ (Die Musik muß ins Mittelmeerische übersetzt werden), schrieb er in sein Notizheft. Da er selbst, wohl oder übel, nicht auch noch diese Aufgabe leisten konnte, setzte er - in der Nähe - Heinrich Köselitz ein, den er zum maestro Pietro Gasti stilisierte, und - in der Ferne - erwählte er Georges Bizet mit seiner Oper Carmen als seinen Hofmusikus [...] Die Frage ist, warum er den großen Komponisten ausließ, ,verdrängte‘, den alle Welt als südlichen Antipoden Wagners begriff und feierte, an der Spitze Jacob Burckhardt: Giuseppe Verdi [...] Verdi gehörte jedermann, Bizet war seine Entdeckung, genauer: er ernannte ihn zum Gegenkaiser.“31

Zu einer ,Mittelmeerakademie‘, in der Nietzsche seine Jünger um die Philosophie Zarathustras und die Musik Bizets geschart hätte, ist es zu seinen Lebzeiten nicht gekommen. Dass er seine kosmopolitische Kolonie im Midi nicht realisieren kann, dafür aber seine Schwester und ihr Schwager ,sehr stolz und glücklich über ihre nunmehr vollendete Besitzergreifung“ (Nr. 472, KGB III/6, 74), der nationalsozialistischen und antisemitischen Kolonie Nueva Germania in Paraguay, sind, muss ihn geärgert haben.

Knapp zwei Jahre vor seinem Zusammenbruch wohnt Nietzsche in Nizza einem „Ereignis“, das andere eine Katastrophe genannt hätten, bei. ${ }^{32}$ Am Aschermittwoch 1887 bebt in Nizza die Erde. Tausende sind obdachlos, viele Häuser, darunter die Pension de Genève, in der zwei seiner Werke entstanden sind, steht nicht mehr (vgl. Nr. 812, KGB III/5, 38). Das Beben sei nur halb so schlimm gewesen, versichert er seiner Mutter, an der gesamten Küste seien nicht mehr als tausend Menschenleben zu beklagen (vgl. Nr. 811, KGB III/5, 36). An seinen Freund Franz Overbeck schreibt er, dass er nach seiner „Inspektionstour“ kurz nach dem Beben gut geschlafen habe und dass das Wetter im übrigen herrlich sei (vgl. Nr. 808, KGB III/5, 33). Selbst an jenem Morgen danach, als Nizza einem Tollhaus geglichen habe, habe er mit „,großer Gemüthsstille“ in seinen Zimmer gearbeitet und habe sogar vergessen, das „Ereigniß des Tages“ in einem Brief zu erwähnen (Nr. 809, KGB III/5, 33 f.): „Bisher bin ich kalt-

31 Werner Ross, Der ängstliche Adler, 738.

32 Vgl. Kurt Freiherr von Witzleben, Das Erdbeben an der Riviera, in: Illustrierte Zeitungvom 1. 3. 1887. 
blütig genug dabei geblieben und habe mitten unter tollgewordenen Tausenden mit dem Gefühl und der kalten Neugierde gelebt [...] Das Plötzliche, das imprévu hat seine Reize“ (Nr. 814, KGB III/5, 42).

Die kalte Neugierde ist die andere Seite des Rausches und der stillstehenden Zeit, die Nietzsche im Midi erfährt. Vor allem die Szene des Zarathustra, als der Schlange der Kopf abgebissen wird, ist für die französischen und späten deutschen Jünger wichtig geworden. Die Utopie eines von der Diktatur der Ratio befreiten intensiv lebenden Menschen verbindet sich in besonderer Weise mit dem Ort Midi. ${ }^{33}$ Er gibt das Geheimnis von „Mittag und Ewigkeit“ (NL 11[195], KSA 9, 519), so heißt es in den nachgelassenen Fragmenten aus dem Jahre 1881, preis.

\section{Der Mittag: Zarathustra}

In seinem ersten Nizza-Winter schreibt Nietzsche das dritte Buch des Zarathustra, welches das berühmte Rätselbild enthält, in dem der Hirte der Schlange den Kopf abbeißt. Die Passage hat eine Unzahl von Deutungen hervorgerufen. Als Kopflosigkeit ist das Bild für die französische Philosophie zentral geworden, wie es bereits Acéphale, der Titel der Zeitschrift des Collège de sociologie, zeigt. Das dritte Buch entsteht als Sanktus Januarius-Ereignis (Gennaro ist der blutende androgyne Heilige von Neapel): In einer eruptiven Inspiration gelingt Nietzsche nicht der Gedanke der ewigen Wiederkehr selbst (er geht auf die frühere Begegnung mit dem Surlei-Felsen im Gebirge zurück), sondern die Umsetzung des Gedankens, seine sprachliche Entäußerung und die entsprechende Lebensführung. So kann er sich zum effizienten Religionsstifter erklären, der eine Lehre verkündet. Diese Lehre benötigt einen entsprechenden Stil. Das Schreiben löst einen Stau. Nietzsche entkommt momenthaft der Diktatur des wissenden Ich.

Nun beansprucht Nietzsche für seine zwischen Literatur und Philosophie changierenden Texte, seine poèmes en prose, eine physiologische Wahrhaftigkeit, eine leibliche Vernunft, eine Probe im Leben. Und hier hat der Mittelmeerraum seinen Platz. Der Zarathustra will ein Text der Gesundung, der Heilung sein. Die Mittelmeerlandschaft wird Ort der frugalen gesunden Lebensführung, in der sich die Lebenskräfte in ein Werk der Lebensverherrlichung ergießen.

Im Sanctus Januarius-Kapitel in Die Fröhliche Wissenschaft (FW, KSA 3, 546) kleidet Nietzsche das Geheimnis des Jasagens zum Leben in das Bild der Welle. In eine Woge des Rausches überrollen die Lebenskräfte - Begehren, Liebe, Raub, Eroberung, Schönheit - alle Moral. Der ewige Fortgang überträgt sich in einer fließenden

33 Vgl. Florian Schneider, Zur Ästhetik der Landschaft bei Nietzsche, in: Clemens Pornschlegel, Martin Stingelin (Hg.), Nietzsche and France. Nietzsche und Frankreich, New York, Berlin 2009, 449-466. 
Sprache. Szenen des Meeres und der Berge, die im Zarathustra wie in Nizza eng beieinander liegen und alternieren, lokalisieren den Gedanken eher in der Höhe, die Praxis am und im Meer. Dabei verwischen sich die Grenzen zwischen den Geschlechtern. Nietzsche ist mit dem Gedanken schwanger, mit dem er im Zarathustra niederkommt.

Im dritten Buch des Zarathustra setzt Nietzsche mit der Liebe ein, die der Moral entgegengesetzt ist. Die Liebe schließt alles Leben ein. „Jedes Ungethüm wolltest du noch streicheln. [...] Die Liebe zu allem, wenn es nur lebt!“ (KSA 4, 196). Darauf folgt das Kapitel Vom Gesicht und Räthsel. Zarathustra predigt auf einem Schiff. Er breitet das Gleichnis aus, das die Lehre von der ewigen Wiederkehr des Gleichen enthält. Am Thorweg befreit er sich von dem Zwerg, den er getragen hat: „Zwei Wege kommen hier zusammen: die gieng noch Niemand zuende“ (ebd., 199). Jeder währt eine Ewigkeit. Sie kommen zusammen im Tor, das den Namen „Augenblick“ trägt. „Von diesem Thorwege läuft eine lange ewige Gasse rückwärts, hinter uns liegt eine Ewigkeit [...] Und wenn Alles schon dagewesen ist: was hältst du Zwerg von diesem Augenblick? Muss auch dieser Thorweg nicht schon - dagewesen sein“ (ebd., 199). Wenn der Weg „alle kommenden Dinge nach sich zieht“, zieht er „Also sich selbst noch“ nach sich (ebd., 200). Die Wege widersprechen sich und kommen doch zusammen.

Hier wird der Ekel Nietzsches am abendländischen Denken, am jüdisch-christlichen Entwurf, an der Metaphysik, am Humanismus laut. Es ist ein Ekel an Endlichkeit, an der einzigen widerspruchslosen linearen Geschichtsbewegung, welche die Herrschaft der ausschließenden Vernunft durchzusetzen vermeint. Vom Standpunkt des Logos (wahr/falsch, gut/böse, schön/hässlich) sind alle Unentscheidbarkeiten, Widersprüchlichkeiten, alle tänzerischen Zweideutigkeiten widerwärtig. Dies ist der Standpunkt des Ekels. ${ }^{34}$ Zarathustra ist dem modernen, im Zeitpfeil des Fortschritts lebenden Menschen, wie auch der wiederkäuenden Kuh, die noch einmal dasselbe verdaut, überlegen. Die Wiederkehr des Gleichen ist nicht die Wiederkehr desselben.

Zarathustra saugt den Ekel auf, um sich von ihm zu befreien. Allerdings hat er noch Angst, sich dem ewigen Kreislauf, dem der Jahreszeiten, von Werden und Vergehen, von Aufbau und Zerstörung ganz zu überlassen. Dies ist im folgenden Rätsel ins Bild gesetzt. Zarathustra trifft auf „einen jungen Hirten [sich] windend, würgend, zuckend, verzerrten Anlitzes, dem eine schwarze schwere Schlange aus dem Munde hieng. Sah ich je so viel Ekel und bleiches Grauen auf Einem Antlitze? Er hatte wohl geschlafen? Da kroch ihm die Schlange in den Schlund - da biss sie sich fest. Meine Hand riss die Schlange und riss: - umsonst! sie riss die Schlange nicht aus dem Schlunde. Da schrie es aus mir: ,Beiss zu! Beiss zu! Den Kopf ab! Beiss zu! - so schrie es aus mir, mein Grauen, mein Hass, mein Ekel, mein Erbarmen, all mein Gutes und Schlimmes schrie mit Einem Schrei aus mir. - Ihr Kühnen um mich! Wer ist der

34 Vgl. Werner Hamacher, Pleroma, in: Ders., Georg Wilhelm Friedrich Hegel. Der Geist des Christentums, 306-318. 
Hirt, dem also die Schlange in den Schlund kroch? Wer ist der Mensch, dem alles Schwerste, Schwärzeste in den Schlund kriechen wird? - Der Hirt aber biss, wie mein Schrei ihm rieth; er biss mit gutem Bisse. Weit weg spie er den Kopf der Schlange -: und sprang empor. - Nicht mehr Hirt, nicht mehr Mensch, - ein Verwandelter, ein Umleuchteter, welcher lachte!“" (Ebd., 201 f.) Der Hirte hat mit dem Kopf der Schlange seine Angst abgebissen. Der Hirte und Zarathustra sind austauschbar. Beide befreien sich von ihrer Angst. Dies macht eine spätere Passage im dritten Buch deutlich: „Der grosse Überdruss am Menschen - der würgte mich und war mir in den Schlund gekrochen: und was der Wahrsager wahrsagte: ,Alles ist gleich, es lohnt sich Nichts, Wissen würgt.‘ [...] Allzuklein der Grösste! - Das war mein Überdruss am Menschen! Und ewige Wiederkunft auch des Kleinsten! - Das war mein Überdruss an allem Dasein!“ (Ebd., 274)

Die Lehre der ewigen Wiederkunft - „Du lehrst, dass es ein grosses Jahr des Werdens giebt, ein Ungeheuer von grossem Jahre: das muss sich, einer Sanduhr gleich, immer wieder von Neuem umdrehn, damit es von Neuem ablaufe und auslaufe“ (ebd., 276) - spricht und schreibt Nietzsche in Nizza aus. Hier dreht er, die Sonne steht im Zenit, im Mittag, im Midi, gewissermaßen die Sanduhr um.

Die halkyonischen Tage glücklicher Ruhe, des Moments des Stillstands, sind anders als die Windstille in der Geschichte der in einen Eisvogel verwandelten Alkyone, in Nizza auch windig. Viele Passagen aus dem Zarathustra lassen sich auch mehr oder weniger platt biographisch lesen. Das Kapitel Von der Seligkeit wider Willen enthält Spuren des mühseligen Existierens in Nizza: „Der Wind blies mir durch's Schlüsselloch und sagte ,Komm!““ (Ebd., 205)

Dieses Kapitel liefert auch Hinweise, um eine andere Dimension der Bedeutung aufzuschließen, die im Bilde des Abbeißens des Schlangenkopfes verborgen ist, nämlich das Phantasma der Kastration. Der Lebensabschnitt am Mittelmeersaum folgt früheren Versuchen Nietzsches, Frauen einzunehmen. Diese Hoffnung hat er nicht mehr. Der Midi ist der Ort der Offenbarung. Aber die Lehre von der ewigen Wiederkehr ist eine düstere schwere Lehre, schwer, die nur forciert in Heiterkeit aufgehoben wird. Zarathustra ist auch ein Text nach der Aufgabe der Heiratspläne, der Dreierpläne mit Andreas-Salomé und Rée, er spiegelt ein Leben ohne Frauen wider. Er ist auch als ein traurig-lüsterner Text eines alternden kränkelnden (impotenten) Mannes lesbar, der nicht zum Zuge kommt. Die Frau lässt sich nicht einnehmen, wie die Wahrheit: „Begehren - das heisst mir schon: mich verloren haben. [...] Aber brütend lag die Sonne meiner Liebe auf mir, im eignen Safte kochte Zarathustra, - da flogen Schatten und Zweifel über mich weg“ (ebd., 205).

Die Feier des Winters dient auch der Abkühlung des Begehrens - „[n]ach Frost und Winter gelüstete mich schon“ (ebd.). Diese doppelte Uneinnehmbarkeit - der Wahrheit und der Frau - eröffnet nun den Weg zu einer Nietzsche-Rezeption, in der die Kritik am Logozentrismus mit derjenigen am Phallogozentrismus zusammenläuft. In Zarathustra heißt es: „Das Glück läuft mir nach. Das kommt davon, dass ich nicht den Weibern nachlaufe. Das Glück aber ist ein Weib“ (ebd., 206). Zwar will alle Lust 
Ewigkeit (vgl. ebd., 286), aber - der begleitende zählende Gongschlag im Gedicht über die Lust hämmert es ein - diese Ewigkeit ist immer schon hinter uns.

Der Hirte bzw. Zarathustra hat der Schlange den Kopf abgebissen. Er hat seine Angst, er hat den Augenblick abgebissen, es gibt keine verbindliche Wahrheit. Das Subjekt entdeckt die Nicht-Identität mit sich selbst, die geschlechtliche einbegriffen. Derrida zeigt in Eperons in einer abweichenden (dekonstruktiven) Lektüre zu Martin Heideggers Nietzsche-Interpretation die Unentscheidbarkeit des Geschlechts. Ausgehend von dem Nietzsche-Zitat „Die Idee [...] wird Weib“ (JGB, KSA 5, 11), arbeitet Derrida heraus, dass Idee wie Weib ,unfasslicher, feiner, verfänglicher“ ${ }^{\text {*35 }}$ werden. Der ,Weiberfeind' Nietzsche, der gegen die geschmacklosen Mannweiber und verunglückten Emanzipierten Mme de Staël und George Sand (JGB, KGW VI.2, 178) wettert, ist auch ein ,Weiberfreund'. Für ihn ist die Lebensfeindlichkeit der Kirche zugleich frauenfeindlich. Nietzsches Stil entspricht der Rätselhaftigkeit der Frau. Er/Sie macht die Opposition zwischen wahr und unwahr nichtig.

Die Hochstimmung im Midi ist indes unerotisch. Die Abkühlung des Begehrens zugunsten einer Identifikation mit dem kopflosen Gott des Rausches führt nur zur Entdeckung des Nichts. Man mag sie mit Nüchternheit, mit Kühle gleichsetzen: „Der Kopf ist im Rausch verkappt - wie mit einer Kappe verblendet. Berauschung ist nicht Kopflosigkeit, sondern maskierte Nüchternheit. In der antiken Mythologie ist einzig der Gott des Rausches selbst, nur Dionysos, kopflos dargestellt worden. Das Ungeheure in Person - oder vielmehr gerade nicht mehr personifiziert, sondern bloss noch Maske, hinter der sich nichts mehr verbirgt... Ganz im Innern des ekstatischen Feuers wird es kühler.“36

Nicht Nüchternheit erwartet indes Nietzsche. Dionysos wird Nietzsche nur um den Preis der Kopflosigkeit des Wahns. Als er Nizza verlässt, um in Turin einen neuen Ort zu finden, den er mit seinen Phantasmen besetzt, ist er ein kranker alter Mann, obwohl noch keine fünfzig, nicht tanzend, sondern hinkend. Die Identifikation mit dem kopflosen Gott im Mittag bezahlt er mit dem Delirium.

\section{Vom genius loci zum Gedächtnisort}

Wenige Spuren hinterlässt Nietzsches Aufenthalt in Nizza. 1888 erscheint sein Name erstmals in der Zeitung Le Petit Niçois auf einer Liste, welche die Ausländer im Ort aufzählt. Man muss allerdings zweimal hinschauen, um ihn darauf zu finden, denn dort wird unser Philosoph versehentlich als „Vlietzche“37 geführt.

35 Jacques Derrida, Sporen. Die Stile Nietzsches, in: Werner Hamacher (Hg.), Nietzsche aus Frankreich, 129-168

36 Gert Mattenklott, Der übersinnliche Leib. Beiträge zur Metaphysik des Körpers, Reinbek 1982, 222.

37 Raoul Mille, Ma Riviera. Chroniques, Nice 2003, 180. 
Sein nach 1890 schlagartig in Frankreich und Deutschland einsetzender Ruhm trägt im Februar 1933 dazu bei, dass in Nizza an der Promenade des Anglais das Kulturzentrum Centre Universitaire Méditerranéen gegründet wird, dessen erster Leiter der französische Lyriker und Philosoph Paul Valéry wird. In seiner Eröffnungsrede betont der Germanist Edouard Spenlé, Rektor der Universität Dijon und NietzscheSpezialist, immer wieder die Bedeutung des großen Umwerters. Zu Ehren des „modernen Troubadours der Provence und des Mittelmeers" wird in der Vorhalle des C.U.M. ein Relief-Bild, ein „médaillon Frédéric Nietzsche“ des französischen Bildhauers Michel de Tarnowsky enthüllt. ${ }^{38}$

In Eze führt ein nach ihm benannter Wanderweg vom Bahnhof am Meer hinauf in das mittelalterliche Dorf. Die vierhundert Meter Höhenunterschied sollen ja, wie eingangs zitiert, Nietzsche zu Stellen im Zarathustra inspiriert haben. Die Gedenktafel, die daran erinnert, ist heute gänzlich zugewachsen. Die rue Ségurane Nummer 38 ziert ein Bronzerelief, auf dem geschrieben steht, dass die Nizza-Winter des Naumburgers hier begonnen haben, ebenso gibt es das bereits erwähnte Relief im C.U.M., 65 Promenade des Anglais. Außerdem ist auf dem Schlossberg eine Terrasse nach Nietzsche benannt. Das sind allesamt Gedenkorte, welche die hivernale Migration des Deutschen nach Frankreich zeigen. Aber hat er je eine mediterrane Mittlerrolle eingenommen? Doch, ein amüsantes Beispiel gibt es. Auch in Nizza setzt nach der Windstille der Wind, Mistral genannt, ein. Auch ihn begrüßt Nietzsche. Die Fröhliche Wissenschaft, 1882 erschienen, bearbeitet und ergänzt Nietzsche 1887 durch die Aufnahme von Gedichten. ${ }^{39}$ Eines davon ist das elfstrophige An den Mistral. Ein Tanzlied, das er am 22. November 1884 mit den Worten „ein [...] Tanz für großes Orchester, das gut brüllen und brausen kann“ (Nr. 557, KGB III/1, 558) an Köselitz alias Peter Gast schickt, dessen erste Strophe ich hier wiedergebe: „Mistral-Wind, du Wolken-Jäger, / Trübsal-Mörder, Himmels-Feger, / Brausender, wie lieb ich dich! / Sind wir Zwei nicht Eines Schoosses / Erstlingsgabe, Eines Looses / Vorbestimmte ewiglich?“ (FW, KGW V/2, 333).

Mit dieser Liebeserklärung wandelt sich Nietzsche - wie vormals der Woge - dem Wind an, einem einmaligen und ewiglichen Ereignis - im Midi, in der Provence. Und um was handelt es sich wohl bei folgenden Versen? „Vènt Maïstrau, cassaire de nivo, / Tuaire de malancounié, Escoubaire dou cèu, / Tu que brames, oh! que iéu t’ame! / Sian-ti Pas tuti dous, / Nous-autre, li primadié d'uno memo urigino, / Eternamen astra pèr lou meme destin!“40 Das ist die Übersetzung des Cansoun dansarello Per Lou Mistrau durch den provençalischen Nobelpreisträger Frédéric Mistral aus dem Jahre 1905. Wenn damit Nietzsche nicht nolens volens zum mediterranen Mittler geworden ist.

38 Vgl. Oscar Levy, Nietzsche-Feier in Nizza, in: Das Neue Tage-Buch, 13. März 1937, Wieder abgedruckt in: Oscar Levy, Nietzsche verstehen. Essays aus dem Exil 1913-1937, Berlin 2005, 253 f.

39 Vgl. Giorgio Colli, Nachwort, in: KSA 3, 663.

40 Frédéric Mistral, Per Lou Mistrau. Cansoun dansarello dou celèbre filousofe et pouèto alemand Nietzche [!], Traducho pèr F. Mistral, Archives Municipales de Nice 1905. 